\title{
Effect of Sodium Valproate on Nocturnal Melatonin Sensitivity to Light in Healthy Volunteers
}

\author{
Karen T Hallam', James S Olver' and Trevor R Norman*,' \\ 'Department of Psychiatry, The University of Melbourne, Austin Hospital, Heidelberg, Victoria, Australia
}

\begin{abstract}
Sensitivity of the pineal hormone melatonin to bright light at night has been proposed as a putative marker of bipolar affective disorder. Patients with bipolar disorder have a super-sensitive melatonin response to light. No studies have investigated whether super-sensitivity is due to agents used to treat the illness or is associated with the disorder per se. We investigated the effect of valproate on this phenomenon. Melatonin sensitivity to light was determined on two nights in 12 healthy volunteers (5M, 7F). Between testing nights participants received $200 \mathrm{mg}$ of valproate b.d. for 5 days. Valproate significantly decreased the sensitivity of melatonin to light. On the other hand, valproate had no effect on overall melatonin secretion or dim light melatonin onset. The ability of valproate to decrease the sensitivity of melatonin to light may relate to its therapeutic effect in bipolar disorder - an ability to lengthen circadian period similar to that of lithium.

Neuropsychopharmacology (2005) 30, |400-|404, advance online publication, 20 April 2005; doi:| 0.1038/sj.npp. I 300739
\end{abstract}

Keywords: melatonin; valproate; light sensitivity; bipolar disorder; chronobiology; nocturnal

\section{INTRODUCTION}

Bipolar affective disorder follows an infradian rhythmic pattern, where manic and depressive episodes may alternate over weeks, months (as in rapid cycling bipolar disorder), or years. Multiple disturbances are characteristic of this disorder, and include seasonal mood changes and circadian dysregulation (Cassidy and Carroll, 2002; Jones, 2001; Shapira et al, 2004). These findings led to the hypothesis that bipolar disorder is primarily a disturbance of rhythmic functions (Healy and Waterhouse, 1995; Leibenluft et al, 1996). As the relationship between bipolar disorder and circadian abnormalities appears to be distinct from other psychiatric illnesses, the potential for rhythmic neuroendocrine disturbances as endophenotypic markers (Gottesman and Gould, 2003) of bipolar disorder are being explored.

Of these markers, the photic entrainment of the hormone melatonin is particularly promising. Melatonin plays a key role in regulating circadian rhythms and appears to be the gating mechanism that regulates the circadian system to correlate with external light conditions (the primary circadian zeitgeiber). Further, the synthesis of melatonin

\footnotetext{
*Correspondence: Professor TR Norman, Department of Psychiatry, The University of Melbourne, Austin Hospital, Level 10 Lance Townsend Building, Austin Hospital Studley Road, Heidelberg, Victoria 3084, Australia, Tel: +61039496 55II, Fax: + 610394590821, E-mail: trevorrn@unimelb.edu.au

Received 4 November 2004; revised 16 February 2005; accepted 3 March 2005

Online publication: 8 March 2005 at http://www.acnp.org/citations/ NPP030805040519/default.pdf
}

can be suppressed by exposing participants to bright nocturnal light (Lewy et al, 1980), an effect which is independent of age (Nathan et al, 1999a), season (Nathan et al, 1999c), menstrual oestrus in females (Nathan et al, 1999d), and differences in melatonin amplitude (Nathan et al, 1999a). While some studies show a gender effect in suppression (Monteleone et al, 1995), recent research in this laboratory showed sensitivity was consistent between males and females across various light intensities (Nathan et al, 1997).

As affective illness is associated with circadian abnormalities and seasonal relapse (Shapira et al, 2004; Wehr, 1990; Wehr et al, 1979), melatonin sensitivity to light has been investigated. In patients with bipolar disorder, light has a significant effect in suppressing melatonin secretion compared with control subjects (Lewy et al, 1985, 1981; Nathan et al, 1999b; Nurnberger et al, 2000). This increased sensitivity is not observed in unipolar depression (Cummings et al, 1989) or seasonal affective disorder (Nathan et al, 1999b) and is not state dependent, occurring over manic, depressed, and euthymic phases of the illness (Lewy et al, 1985). Melatonin sensitivity appears to be strongly heritable with the offspring of affected parents having greater sensitivity than offspring of healthy controls (Nurnberger et al, 1988).

Together these findings fulfil many of the desired criteria of an endophenotype of BPAD: association with illness, heritable, and state independent (Gottesman and Gould, 2003). Several questions remain concerning sensitivity, specificity, and the effects of medication on responses observed in patients before melatonin light sensitivity can 
be accepted as an endophenotype. Indeed, some suggestion of the effect of medication on nocturnal melatonin light sensitivity was evident in at least one report (Nurnberger et al, 2000). Among bipolar I patients, the greatest melatonin sensitivity was observed in those free of medication for at least 5 weeks, while those taking lithium alone had melatonin suppression similar to that of controls. Recent research in our laboratory (Hallam et al, 2004) demonstrated that lithium reduces melatonin suppression by light in healthy controls. The effect of other mood stabilizing agents used in the treatment of BPAD remains unknown. Monteleone et al (1997) demonstrated that single doses of valproate acutely suppressed melatonin secretion in healthy volunteers. No studies have investigated the effect of this or other mood stabilizing agents on sensitivity to light or other parameters of the melatonin rhythm.

In this report, we assessed the effect of valproate administration on melatonin sensitivity to light, dim light melatonin onset (DLMO), and the nocturnal secretion of melatonin in healthy volunteers. It was hypothesized that valproate would significantly reduce the sensitivity of melatonin to light and advance melatonin phase in healthy controls. Furthermore, we hypothesized that subchronic valproate treatment may decrease nocturnal melatonin secretion.

\section{METHODS}

\section{Participants}

In total, 12 healthy adult volunteers $(7 \mathrm{~F}, 5 \mathrm{M})$ with a mean age of 22.9 ( $\mathrm{SD} \pm 5.6)$ years provided written informed consent to participate in this study. All participants were free of a personal or family history of psychiatric illness as determined by clinical interview with a psychiatrist (JO) and questionnaires (General health questionnaire (Goldberg and Hillier, 1979), PRIME-MD (Spitzer et al, 1994)). Physical examinations and hematology workup indicated that all participants were in good physical health. Five of seven female participants were using oral contraceptive medications. All participants self-report sleep parameters were normal with sleep onset between 2200 and 2400, average sleep latency $30 \mathrm{~min}$, and normal sleep duration $(8 \pm 1.5 \mathrm{~h})$. In total, 10 participants consumed caffeine daily $(1.4 \pm 1.2$ standard drinks) and were low to moderate alcohol consumers $(4.5 \pm 5$ standard drinks per week). Four participants were cigarette smokers $(13 \pm 8$ per day). The Human Research Ethics Committee of Austin Health Network approved the study.

\section{Procedure}

Participants attended the Melatonin Laboratory in the Department of Psychiatry at the Austin Hospital on two testing nights separated by a 7-day recovery period. At the commencement of each testing night, a canula was placed into the forearm vein of each participant and kept patent through the night with $0.9 \%$ sodium chloride solution. Blood samples were collected at regular intervals between 2000 and 0230. Participants remained seated in recliner chairs and were provided with a standardized meal at 2000. At 2100 hours the background lights were extinguished and the environmental light intensity maintained at less than 8 lux (monitored using a Topcon 3D digital photometer) as measured at each participant's eye level.

On each testing night, a light box was placed in front of the participants to create a full spectrum white light at an intensity of 200 lux at eye level between 2400 and 0100 . McIntyre et al (1989) have demonstrated that both this time period and light intensity are appropriate for evaluating melatonin suppression rates in control subjects. The light box was custom made ( a series of eight $97 \mathrm{~W}$ 'True Light' fluorescent tubes behind a diffuser screen. Participants were positioned directly facing the light box, which was facilitated by positioning a television directly above. Photophobic behavior such as reading or closing the eyes was not permitted and participants were instructed to look directly at the light every few minutes for at least $30 \mathrm{~s}$. The experimenter returned at 5-min intervals during this period to check compliance. Light levels were measured at the corneal level at a horizontal angle of gaze for each individual and recorded to be at the appropriate intensity ( \pm 12 lux $)$ at four times throughout the hour. At 0100 the room was returned to virtual darkness ( $<8$ lux of light). Samples of $10 \mathrm{ml}$ of blood were collected from each participant at 2000, 2100, 2130, 2200, 2230, 2300, 2315, 2330, 2345, 2400, 0015, 0030, $0045,0100,0130,0200$, and $0230 \mathrm{~h}$, placed in lithium heparin tubes and the plasma separated by centrifuge and stored at $-20^{\circ} \mathrm{C}$ for analysis.

Melatonin analysis was performed by the Department of Obstetrics and Gynaecology at the University of Adelaide. The melatonin assay utilized reagents from Buhlmann Laboratories (Switzerland) as previously described (Voultsious et al, 1997). Briefly, 250-500 $\mu$ l of plasma was added to the prewashed C18 columns and sequentially washed with $10 \%$ methanol, hexane, and the melatonin finally eluted with pure methanol. After solvent evaporation, the residue was re-constituted in $1 \mathrm{ml}$ of buffer (phosphate-buffered saline, $0.1 \mathrm{M}, \mathrm{pH} 7.4$, containing $0.5 \%$ bovine serum albumin) and two $400 \mu \mathrm{l}$ aliquots subjected to radioimmunoassay. The assay range was between 4.3 and $430 \mathrm{pM}$ with intra- and interassay coefficients of variation $<10$ and $<14 \%$, respectively, across the range of the standard curve. The antibody for the assay is highly specific and demonstrates $<1 \%$ crossreactivity with other compounds in the melatonin synthetic pathway. An area under the plasma melatonin profile was calculated using the trapezoidal method (Gibaldi and Perrier, 1975) and Fig $\mathrm{P}^{\mathrm{TM}}$ (Biosoft) processing software. This calculation is used to provide an integrated measure of plasma melatonin concentrations between 2000 and 2400 (before lights). Melatonin suppression between 2400 and 0100 was determined using the following formula:

$$
\begin{aligned}
& \text { percentage suppression } \\
& =\frac{((2345+2400) / 2)-\left(\left(0045 \_0100\right) / 2\right)}{((2345+2400) / 2)} \times \frac{100}{1}
\end{aligned}
$$

At 2 days after the first testing night, each subject was instructed to take a 5-day course of sodium valproate (200 mg b.d. with food starting with an evening dose). On the fifth day, subjects took their final morning dose of 
valproate and returned empty containers on the second testing night. On the second testing night, a valproate level was taken $12 \mathrm{~h}$ after the final dose from each participant (in lithium heparin tubes). Valproate levels were measured using the AxSYM valproic acid assay (a Fluorescence Polarization Immunoassay, FPIA) that utilizes sheep polyclonal antiserum. The assay has a detection range of $4.85-1039.50 \mu \mathrm{mol} / \mathrm{l}$, sensitivity of $0.70 \mu \mathrm{g} / \mathrm{ml}$, CV (precision) $<5 \%$, and crossreactivity of $<10 \%$. Side effects from valproate were mild and infrequent, with four participants reporting mild sedation during the week and one participant reporting increased gastric reflux.

\section{RESULTS}

\section{Plasma Valproate Level}

Plasma valproate measures indicate that all participants had circulating valproate on the second testing night indicating drug compliance. The average valproate level was $204 \mu \mathrm{mol} /$ $1( \pm 63 \mu \mathrm{mol} / \mathrm{l})$, which is less than the recommended therapeutic range $(350-700 \mu \mathrm{mol} / \mathrm{l})$.

\section{Melatonin Suppression by White Light}

Melatonin suppression between 2400 and 0100 was determined for each participant on each testing night (see Table 1). Changes in individual melatonin suppression for the two testing nights were compared using a paired samples $t$-test. The results indicated that there was a significant difference in melatonin suppression by dim white light between the control and valproate conditions, $t$ $(1,11)=2.751, \quad p=0.019$. A calculation of Cohen's $d$ indicates an effect size of 0.709 .

\section{Dim Light Melatonin Onset}

DLMO was calculated based on the work of Lewy et al (1999). Lewy recommends using a DLMO value between 4 and $6(\mathrm{pg} / \mathrm{ml})$. This study therefore utilized a DLMO (4 pg/ $\mathrm{ml}$ or $17.2 \mathrm{pmol} / \mathrm{l}$ ) as a sensitive yet reliable marker to the onset of melatonin. In this study, DLMO was calculated as the first time period where melatonin levels increased above and remained above this threshold. The DLMO results are presented in Table 1. There was a nonsignificant trend for DLMO to delay across testing night, $t(1,11)=-1.326$, $p=0.212$.

Table I Changes in Melatonin Suppression by Light, DLMO, and AUC Values Between Control and Valproate Treatment Nights $( \pm$ SEM)

Effect of valproate on other melatonin parameters

\begin{tabular}{llc}
\cline { 2 - 3 } & Control night & After subchronic valproate \\
\hline Suppression $(\%)$ & $35.33( \pm 8.44 \%)$ & $12.57( \pm 10.01 \%)^{*}$ \\
DLMO $(\mathrm{h})$ & $2205( \pm 8.1 \mathrm{~min})$ & $2227( \pm 13.5 \mathrm{~min})$ \\
AUC $(\mathrm{pM} / / / \mathrm{h})$ & $1144( \pm 268)$ & $997( \pm 150)$ \\
\hline
\end{tabular}

*Significantly decreased at $p=0.019$.

\section{Average Melatonin Secretion}

Area under the curve estimates were determined between the hours of 2100 and 2400 (ie a nine point estimate based on the acrophase). A paired samples $t$-test was performed on melatonin AUC across testing night and the results revealed that the mean melatonin AUC value was decreased, but this change was not statistically significant, $t(1,11)=$ $0.629, p=0.542$. Table 1 provides mean AUC values across testing night.

\section{DISCUSSION}

This study demonstrates that subchronic administration of valproate significantly reduced the sensitivity of nocturnal melatonin secretion to light in healthy volunteers. In this respect, valproate and lithium share a common biological effect as we have previously reported similar effects in volunteers taking lithium (Hallam et al, 2004). Both sets of data are in accord with a trend noted in bipolar patients by Nurnberger et al (2000). They showed that in drug-free bipolar patients, the effect of light on nocturnal melatonin secretion was greater than that in patients treated with lithium. It is not clear why this effect occurs. It may be that both drugs phase delay the melatonin rhythm and lower sensitivity to light as a consequence. Several factors do not accord with this explanation. First in this study, there was on average only a 20 -min delay in DLMO by valproate. On the other hand, lithium has been shown to significantly delay the melatonin rhythm in the chick pineal, but only at concentrations in the toxic range in humans $(2 \mathrm{nmol} / \mathrm{l})$ (Pablos et al, 1994). Even if such phase delays do occur, they cannot be the explanation for the decreased melatonin sensitivity to light. We have previously shown that melatonin is equally sensitive to the effects of light across the night - on the rising part of the melatonin time curve, at the peak or during the falling part of the curve in the early morning (McIntyre et al, 1989). Thus, phase shifts are unlikely to alter melatonin sensitivity to light. A second possibility is that an overall reduction in the amount of melatonin secreted may desensitize the system. Although valproate administration was associated with a reduced secretion of nocturnal melatonin, the reduction was not statistically significant in this study. Furthermore, we have previously shown that the sensitivity of melatonin to light is independent of melatonin concentration immediately prior to the onset of light (Nathan et al, 1999a). Finally, while this study indicates valproates primary effects are on melatonin sensitivity to light, the drug may also affect other parameters of the melatonin rhythm not tested in this study.

Lewy et al (1985) suggest that increased light sensitivity may lead to the circadian phase advances observed in bipolar disorder. Studies in rapid cycling bipolar disorder indicate that the timing of the melatonin peak is vital with morning, but not noon light tending to induce hypomania in some patients (Kripke, 1991; Leibenluft et al, 1995). Two groups have recently demonstrated that lengthened sleep periods and enforced darkness ameliorate the symptoms of rapid cycling bipolar disorder (Wehr et al, 1998; WirzJustice et al, 1999). We propose that lithium and valproate may share a common mechanism of action in decreasing melatonin sensitivity to light, leading long-term to the

Neuropsychopharmacology 
normalization of the circadian phase advance that has been observed in patients with bipolar disorder. This contention is supported by the findings that under normal circumstances, dawn light is sufficient to entrain the circadian rhythm (Danilenko et al, 2000), but lithium is associated with a delay in the fall of plasma melatonin normally observed at dawn (Nair et al, 1984). Clearly, further empirical research is required in drug-free bipolar patients to determine the chronobiological impact of these drugs in the patient population as opposed to control subjects.

The finding that chronic valproate treatment did not alter overall melatonin levels is in contrast to acute results where valproate decreased melatonin concentrations (Monteleone et al, 1997). It was proposed that valproate affects melatonin synthesis by its ability to enhance GABA signalling. It is of note that other GABA agonists, such as benzodiazepines, also acutely suppress nocturnal melatonin synthesis (McIntyre et al, 1988).

The contrast between acute effects of valproate noted by Monteleone et al, and the subchronic effects noted in the present study might be explained by methodological factors (small sample size or high interindividual variability) or receptor adaptation. In relation to receptor adaptation, other drugs have different acute and chronic effects on melatonin secretion. For example, desipramine acutely increases melatonin synthesis (Franey et al, 1986; Kennedy and Brown, 1992; Skene et al, 1994) but after chronic treatment, melatonin is no longer elevated (Kennedy and Brown, 1992), an activity possibly associated with pinealocyte noradrenergic receptor downregulation. Pharmacokinetic effects of the drug do not account for the changes in melatonin concentration with recent evidence indicating melatonin is primarily metabolized by the cytochrome P4501A2 enzymes (Härtter et al, 2000, 2003), while valproate has no discernable effect on the activity of this enzyme (Wen et al, 2001). The results of this study suggest that decreases in melatonin after acute but not chronic valproate treatment may be associated with acute GABAergic activity of the compound on the pinealocyte, which adapts after subchronic treatment.

In healthy volunteers, both lithium (Hallam et al, 2004) and valproate decrease melatonin sensitivity to light. While the aim of this research was to further clarify the usefulness of melatonin sensitivity analysis for the diagnosis of bipolar disorder, it is important to note that this study investigated the effect of valproate treatment on volunteers and that melatonin sensitivity changes associated with valproate treatment may differ in patients and controls. We propose that these results, when analyzed with those from Nurnberger et al (2000) that medication-free patients appeared to have the highest sensitivity, may reflect important effects of medications, namely the normalization of melatonin supersensitivity to light. The mechanism of this effect is not clear; however, in the context of recent proposals that mood stabilizing drugs share common biological effects, such as depletion of inositol and GSK-3 (Harwood and Agam, 2003; Jope, 2003; Williams et al, 2002), an intracellular mediated effect could be hypothesized. Further research is necessary to evaluate such a proposal but, given the complexity of neurotransmitter pathways mediating bright light induced alterations of nocturnal melatonin synthesis; these results have at least heuristic value. These data provide further support for melatonin sensitivity to light as an endophenotypic marker for the diagnosis of bipolar disorder.

\section{ACKNOWLEDGEMENTS}

We would like to thank Denovan Begg (Latrobe University) for assistance with data collection and Athena Voultsious (Department of Gynaecology and Obstetrics, The University of Adelaide) for assistance with melatonin analysis. We would also like to acknowledge Sara, Mandy, Rebecca, Hilde, Lee, and Cassie (Latrobe University) for their assistance on testing nights. No authors have any financial disclosures or conflict of interest that have biased the content of this communication.

\section{REFERENCES}

Cassidy F, Carroll BJ (2002). Seasonal variation of mixed and pure episodes of bipolar disorder. J Affect Disord 68: 25-31.

Cummings MA, Berga SL, Cummings KL, Kripke DF, Haviland MG, Golshan S et al (1989). Light suppression of melatonin in unipolar depressed patients. Psychiatry Res 27: 351-355.

Danilenko KV, Wirz-Justice A, Krauchi K, Weber JM, Terman M (2000). The human circadian pacemaker can see by dawn's early light. J Biol Rhythms 15: 437-446.

Franey C, Aldhous ME, Burton S, Checkley S, Arendt J (1986). Acute treatment with desipramine stimulates melatonin and 6-sulfatoxymelatonin production in man. $\mathrm{Br} \mathrm{J}$ Clin Pharmacol 22: 73-79.

Gibaldi M, Perrier D (1975). Pharmacokinetics. M Dekker: New York.

Goldberg DP, Hillier VF (1979). A scaled version of the General Health Questionnaire. Psychol Med 9: 139-145.

Gottesman II, Gould TD (2003). The endophenotype concept in psychiatry: etymology and strategic intentions. Am J Psychiatry 160: 636-645.

Hallam KT, Olver JS, Horgan JE, McGrath C, Norman TR (2004). Low doses of lithium carbonate reduce melatonin light sensitivity in healthy volunteers. Int J Neuropsychopharmacol doi: $10.1017 / S 1461145704004894$.

Härtter S, Grozinger M, Weigmann H, Roschke J, Hiemke C (2000). Increased bioavailability of oral melatonin after fluvoxamine coadministration. Clin Pharmacol Therap 67: 1-6.

Härtter S, Nordmark A, Rose D, Bertilsson L, Tybring G, Laine K (2003). Effects of caffeine intake on the pharmacokinetics of melatonin, a probe drug for CYP1A2 activity. $\mathrm{Br} J$ Clin Pharmacol 56: 679-682.

Harwood AJ, Agam G (2003). Search for a common mechanism of mood stabilizers. Biochem Pharmacol 66: 179-189.

Healy D, Waterhouse JM (1995). The circadian system and the therapeutics of the affective disorders. Pharmacol Therap 65: 241-263.

Jones SH (2001). Circadian rhythms, multilevel models of emotions and bipolar disorder - an initial step towards integration. Clin Psychol Rev 21: 1193-1209.

Jope RS (2003). Lithium and GSK-3: one inhibitor, two inhibitory actions, multiple outcomes. Trends Pharmacol Sci 24: 441-443.

Kennedy SH, Brown GM (1992). Effects of chronic antidepressant treatment with adinazolam and desipramine on melatonin output. Psychiatry Res 43: 177-185.

Kripke DF (1991). Timing of phototherapy and occurrence of mania. Biol Psychiatry 29: 1156.

Leibenluft E, Feldman-Naim S, Turner EH, Schwartz PJ, Wehr TA (1996). Salivary and plasma measures of dim light melatonin onset (DLMO) in patients with rapid cycling bipolar disorder. Biol Psychiatry 40: 731-735. 
Leibenluft E, Turner EH, Feldman-Naim S, Schwartz PJ, Wehr TA, Rosenthal NE (1995). Light therapy in patients with rapid cycling bipolar disorder: preliminary results. Psychopharmacol Bull 31: 705-710.

Lewy AJ, Cutler NL, Sack RL (1999). The endogenous melatonin profile as a marker for circadian phase position. J Biol Rhythms 14: $227-236$.

Lewy AJ, Nurnberger Jr JI, Wehr TA, Pack D, Becker LE, Powell RL et al (1985). Supersensitivity to light: possible trait marker for manic-depressive illness. Am J Psychiatry 142: 725-727.

Lewy AJ, Wehr TA, Goodwin FK, Newsome DA (1981). Manicdepressive patients may be supersensitive to light (letter). Lancet 1: 383-384

Lewy AJ, Wehr TA, Goodwin FK, Newsome DA, Markey SP (1980). Light suppresses melatonin secretion in humans. Science 210: $1267-1269$.

McIntyre IM, Burrows GD, Norman TR (1988). Suppression of plasma melatonin by a single dose of the benzodiazepine alprazolam in humans. Biol Psychiatry 24: 108-112.

McIntyre IM, Norman TR, Burrows GD, Armstrong SM (1989). Human melatonin response to light at different times of the night. Psychoneuroendocrinology 14: 187-193.

Monteleone P, Esposito G, La Rocca A, Maj M (1995). Does bright light suppress nocturnal melatonin secretion more in women than men? J Neural Transm 102: 75-80.

Monteleone P, Tortorella A, Borriello R, Natale M, Cassandro P, Maj M (1997). Suppression of nocturnal plasma melatonin levels by evening administration of sodium valproate in healthy humans. Biol Psychiatry 41: 336-341.

Nair NPV, Hariharasubramanian N, Pilapil C (1984). Effect of lithium on circadian rhythm of plasma melatonin in normal men. IRCS Med Sci 12: 854-861.

Nathan PJ, Burrows GD, Norman TR (1997). The effect of dim light on suppression of nocturnal melatonin in healthy women and men. J Neural Transm 104: 643-648.

Nathan PJ, Burrows GD, Norman TR (1999a). The effect of age and pre-light melatonin concentration on the melatonin sensitivity to dim light. Int Clin Psychopharmacol 14: 189-192.

Nathan PJ, Burrows GD, Norman TR (1999b). Melatonin sensitivity to dim white light in affective disorders. Neuropsychopharmacology 21: 408-413.

Nathan PJ, Burrows GD, Norman TR (1999c). Melatonin sensitivity to dim white light in different seasons. Hum Psychopharmacol 14: $53-58$.

Nathan PJ, Norman TR, Burrows GD (1999d). Effect of the menstrual cycle stage on the melatonin suppression by dim white light. Psychoneuroendocrinology 24: 193-200.
Nurnberger Jr JI, Adkins S, Lahiri DK, Mayeda A, Hu K, Lewy A et al (2000). Melatonin suppression by light in euthymic bipolar and unipolar patients. Arch Gen Psychiatry 57: 572-579.

Nurnberger Jr JI, Berrettini W, Tamarkin L, Hamovit J, Norton J, Gershon E (1988). Supersensitivity to melatonin suppression by light in young people at high risk for affective disorder. A preliminary report. Neuropsychopharmacology 1: 217-223.

Pablos MI, Santaolaya MJ, Agapito MT, Recio JM (1994). Influence of lithium salts on chick pineal gland melatonin secretion. Neurosci Lett 174: 55-57.

Shapira A, Shiloh R, Potchter O, Hermesh H, Popper M, Weizman A (2004). Admission rates of bipolar depressed patients increase during spring/summer and correlate with maximal environmental temperature. Bipolar Disord 6: 90-93.

Skene DJ, Bojkowski CJ, Arendt J (1994). Comparison of the effects of acute fluvoxamine and desipramine administration on melatonin and cortisol production in humans. $\mathrm{Br} \mathrm{J} \mathrm{Clin}$ Pharmacol 37: 181-186.

Spitzer RL, Williams JB, Kroenke K, Linzer M, de Gruy FV, Hahn SR et al (1994). Utility of a new procedure for diagnosing mental disorders in primary care. The PRIME-MD 1000 study. JAMA 272: 1749-1756.

Voultsious A, Kennaway DJ, Dawson D (1997). Salivary melatonin as a circadian phase marker: validation and comparison with plasma melatonin. J Biol Rhythms 12: 457-466.

Wehr TA (1990). Effects of sleep and wakefulness in depression and mania. In: Montplaisir J, Godbout R (eds). Sleep and Biological Rhythms. Oxford University Press: London. pp 42-86.

Wehr TA, Turner EH, Shimada JM, Lowe CH, Barker C, Leibenluft E (1998). Treatment of a rapid cycling bipolar patient by using extended bed rest and darkness to stabilize the timing and duration of sleep. Biol Psychiatry 43: 822-828.

Wehr TA, Wirz-Justice A, Goodwin FK, Duncan W, Gillin JC (1979). Phase advance of the circadian sleep-wake cycle as an antidepressant. Science 206: 710-713.

Wen X, Wang JS, Kivisto KT, Neuvonen PJ, Backman JT (2001). In vitro evaluation of valproic acid as an inhibitor of human cytochrome $P 450$ isoforms: preferential inhibition of cytochrome P450 2C9 (CYP2C9). Br J Clin Pharmacol 52: 547-553.

Williams RS, Cheng L, Mudge AW, Harwood AJ (2002). A common mechanism of action for three mood-stabilizing drugs. Nature 417: 292-295.

Wirz-Justice A, Quinto C, Cagochen C, Werth E, Hock C (1999). A rapid-cycling bipolar patient treated with long nights, bedrest and light. Biol Psychiatry 45: 1075-1077. 